\title{
Left upper lobectomy after coronary artery bypass grafting
}

\author{
Benjamin Wei, MD, Brett Broussard, MD, Ayesha Bryant, MD, Paul Linsky, MD, \\ Douglas J. Minnich, MD, and Robert J. Cerfolio, MD, MBA
}

\section{ABSTRACT}

Objective: Left upper pulmonary lobectomy or segmentectomy after coronary artery bypass grafting $(\mathrm{CABG})$ risks injury to the grafts. We reviewed our experience.

Methods: This is a retrospective review of a prospective database from 1 surgeon, of patients who underwent left upper lobectomy after having previous CABG.

Results: Between June 1998 and June 2014, a total of 2207 patients underwent lobectomy by 1 surgeon; 458 (21\%) had a left upper lobectomy, and $28(6.1 \%)$ had had a previous CABG. Twenty-seven patients $(96.4 \%)$ had a left internal mammary artery (LIMA) used for the bypass. Twenty-six patients $(96.2 \%)$ had significant adhesions between their lung and the bypass grafts. Of patients who had a LIMA graft, $25(92.6 \%)$ had the left upper lobe completely dissected free from their grafts, whereas 2 patients $(7.1 \%)$ had a sliver of their lung left on the grafts. No patient had a postoperative myocardial infarction, and 30-day and 90 -day survival rates were both $100 \%$. All patients had a curative resection, and all had complete thoracic lymphadenectomy.

Conclusions: Left upper lobectomy after CABG, in patients with previous CABG and LIMA grafting, is safe. Usually the entire lung can be safely mobilized off the bypass grafts; if needed, a small sliver of lung can be left on the grafts. A curative resection is possible with minimal perioperative cardiac morbidity, and excellent 30- and 90-day mortality. (J Thorac Cardiovasc Surg 2015;150:531-5)

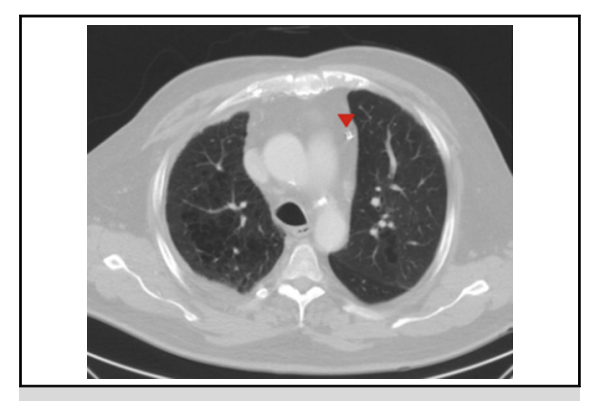

A computed tomography scan with intravenous contrast demonstrates the position of a left internal mammary artery graft (red arrow).

\section{Central Message}

Left upper lobectomy after CABG can be done safely and with minimal complications.

\section{Perspective}

A previous CABG is considered risky in the setting of left upper lobectomy. This study describes our preoperative evaluation, intraoperative conduct, and postoperative results in this situation. Our data show that complete resection of the lung off a LIMA graft can be achieved with a high level of safety, and that previous CABG should not be considered a contraindication for left upper lobectomy.

See Editorial Commentary page 536.
Previous coronary artery bypass grafting (CABG) can complicate lung surgery, owing to the stimulation of adhesion formation and the location of bypass grafts in the chest. Specifically, the presence of a pedicled left internal mammary artery (LIMA) used for coronary bypass poses an especially risky situation for left upper lobectomies, because many patients are graft dependent. When used for coronary bypass grafting, the LIMA is

\footnotetext{
From the Division of Cardiothoracic Surgery, University of Alabama at Birmingham Medical Center, Birmingham, Ala.

Received for publication March 25, 2015; revisions received May 15, 2015; accepted for publication May 23, 2015; available ahead of print July 3, 2015.

Address for reprints: Robert J. Cerfolio, MD, MBA, Division of Cardiothoracic Surgery, Chief of Thoracic Surgery, University of Alabama at Birmingham, 703 19th St S, ZRB 739, Birmingham, AL 35294 (E-mail: rcerfolio@uabmc.edu). $0022-5223 / \$ 36.00$

Copyright (C) 2015 by The American Association for Thoracic Surgery http://dx.doi.org/10.1016/j.jtcvs.2015.05.066
}

normally dissected out as a pedicle, leaving its proximal extent in situ, and anastomosed to the left anterior descending artery. The LIMA can be injured during subsequent thoracic surgery anywhere along its course from the apex of the chest to the target vessel on the heart. Injury can result in hypotension, myocardial ischemia, infarction, and death.

The safety of left upper lobectomy after previous CABG has been described in only a single case series involving thoracoscopic lobectomies, and scattered case reports. ${ }^{1-3}$ The purpose of our study is to describe our experience with left upper lobectomy after $\mathrm{CABG}$, with open versus minimally invasive surgery. This series is the largest published, to date, of patients undergoing left upper lobectomy after CABG.

\section{METHODS}

This retrospective, cohort study used a database of prospectively collected data from 1 general thoracic surgeon, evaluating patients undergoing left upper lobectomy, via either thoracotomy or a robotics approach. 


\section{Abbreviations and Acronyms \\ $\mathrm{CABG}=$ coronary artery bypass grafting \\ LIMA $=$ left internal mammary artery}

Port placement and conduction of a robotically assisted lobectomy has been described previously. ${ }^{4}$ Previous CABG had been done via median sternotomy and with an on-pump strategy. Preoperative studies included computed tomography scans with intravenous contrast, stress testing, and in selected patients with ischemia during stress testing, left heart catheterization. Intraoperatively, the left upper lobe was preferentially mobilized off the LIMA graft.

With thoracotomy, a combination of blunt dissection with suction devices, and sharp dissection with Metzenbaum scissors, was done. With the robotic approach, a Cadiere grasper and bipolar curved blunt tip dissector were used to free the lung from the graft. Dissection of the left upper lobe is generally begun at the lingula, first taking it off the diaphragm, working from inferior to superior, and using the clips found on the LIMA graft to help identify it. Alternatively, if this dissection was deemed unsafe, and the lung cancer was not in the anterior segment of the left upper lobe or lingula, a small sliver of lung was divided from the rest of the left upper lobe with a stapler, and was left attached to the graft. Before 2005, most of these patients had arterial lines and Foley catheters placed, as was standard for all lobectomy patients. After 2005, invasive monitoring was used on a case-by-case basis at the discretion of the surgeon and anesthesiologist.

Information was obtained through hospital databases and medical records. Data were exported from Excel (Microsoft Corporation, Redmond, Wash) to SAS, version 9.1 (SAS Institute, Inc, Cary, NC). Descriptive statistics were used to estimate the frequency of categoric variables and the median of the continuous variables. The University of Alabama at Birmingham's Institutional Review Board approved this protocol, as well as the prospective database used to collect information for this study. Individual consent was waived for patient inclusion in this study; however, consent was required, and obtained, to enter patient data in the prospective database.

\section{RESULTS}

Between June 1998 and November 2014, a total of 2207 patients underwent lobectomy, performed by 1 surgeon, and $458(21 \%)$ patients had a left upper lobectomy. Of these 458 patients, $28(6.1 \%)$ had had a previous CABG operation; 26 a single previous CABG operation; and $2(7.1 \%)$ had $>1$ previous $\mathrm{CABG}$ operation. Their preoperative characteristics are shown in Table 1 . All but 1 had a LIMA graft. Twenty-four $(86 \%)$ of these patients had undergone a stress test during their preoperative evaluation for surgery; 19 of these patients $(79.2 \%)$ had no evidence of ischemia, and $6(21.4 \%)$ of these had areas of ischemia. Left heart catheterization was performed in these patients as part of the preoperative evaluation for lobectomy. One of these patients underwent coronary stent placement at the same time as the catheterization; the other 5 did not have any coronary disease that justified intervention. Three patients $(10.7 \%)$ received preoperative chemotherapy; $2(7.1 \%)$ received preoperative radiation; and none received preoperative chemoradiation.

Overall, 8 patients $(28.6 \%)$ had an arterial line placed preoperatively, $9(32.1 \%)$ had a central line placed, and
$11(39.3 \%)$ had a Foley catheter placed. Starting in 2006, only a single patient of $15(6.7 \%)$ had either an arterial line or central line placed preoperatively.

Twenty-five patients $(89.2 \%)$ underwent lobectomy via planned thoracotomy; for 2 patients, $(7.1 \%)$ a robotics approach was used initially, requiring conversion to thoracotomy; and 1 patient $(3.6 \%)$ had a complete lobectomy using robotics. The conversion from a robotics approach to thoracotomy was performed in 1 patient secondary to the need to perform pulmonary arterial resection and repair to achieve a curative resection, and in the other patient for bleeding from a pulmonary arterial branch.

Of the 27 patients for whom a LIMA graft was used, 25 $(92.6 \%)$ had the left upper lobe completely mobilized off the LIMA graft, and $2(7.4 \%)$ had a wedge of lung left attached to it. No patients experienced injury to the LIMA graft during the operation. Mean operating time was 1 hour and 34 minutes (range: 65-269 minutes). The curative resection rate was $100 \%$. The median number of lymph nodes resected was 12 . Twenty-six $(92.9 \%)$ patients had lung cancer; 2 (7.1\%) had metastatic renal cell carcinoma. Intraoperative and pathologic details from the series are shown in Table 2.

Median length of hospital stay was 4 days (range: 2-11 days). Morbidity occurred in $12(42.9 \%)$ patients, as described in Table 3. No patients had a perioperative myocardial infarction; $17.9 \%$ had atrial fibrillation. No patients had postoperative bleeding requiring a reoperation. Four patients $(14.3 \%)$ were admitted to the intensive care unit directly from the operating room; 1 patient of the remaining $24(4.2 \%)$ was transferred to the intensive care unit for hypoxia and hypotension after initial recovery in a non-ICU setting. The 30-day and 90-day mortality rates were both $0 \%$.

\section{DISCUSSION}

Left upper lobectomy can be difficult after CABG, especially when patients are graft dependent. Shah and colleagues ${ }^{1}$ have demonstrated the safety of thoracoscopic left upper lobectomy after previous LIMA grafting in 14 patients. In that paper, the authors compared these patients with 276 patients who underwent thoracoscopic left upper lobectomy without previous CABG and showed a similar outcome with no increased perioperative cardiac morbidity. The authors used a strategy of preferentially leaving behind a small wedge of lung parenchyma when it was adherent to the LIMA graft, and their cancer recurrence rate and 5-year overall survival rate were not significantly decreased versus their control groups. Santini and colleagues ${ }^{5}$ reported a case in which they used the LigaSure system (ValleyLab Inc, Boulder, Colo), rather than a stapler, to resect as much of the lung as possible from an intact LIMA graft. 
TABLE 1. Preoperative characteristics of patients undergoing left upper lobectomy after previous CABG

\begin{tabular}{lc}
\hline \multicolumn{1}{c}{ Variable } & Value \\
\hline Patient age (y) & $70.9 \pm 6.6$ \\
Gender, male & $25(89.2)$ \\
History of tobacco use & $28(100)$ \\
Hypertension & $18(64.3)$ \\
Diabetes & $7(25)$ \\
Congestive heart failure & $4(14.3)$ \\
Coronary artery disease & $28(100)$ \\
Atrial fibrillation & $4(14.3)$ \\
Neoadjuvant chemotherapy & $3(10.7)$ \\
Neoadjuvant radiation therapy & $2(7.1)$ \\
Neoadjuvant chemoradiation & $0(0)$ \\
DLCO (\% predicted) & $78.2 \pm 22.0$ \\
FEV1 (\% predicted) & $69.8 \pm 19.9$ \\
\hline
\end{tabular}

Values are presented as raw number $(\%)$, or mean \pm SD. $D L C O$, Diffusion capacity of lung for carbon monoxide; $F E V I$, forced expiratory volume in 1 second.

Based on our experience, we favor complete resection, if possible, especially if the tumor is anteriorly located in the left upper lobe. Our study shows that the left upper lobe can be safely dissected off of the LIMA pedicle with minimal additional risk. In our series, $92 \%$ of patients had this done, and none had an intraoperative LIMA injury, perioperative myocardial infarction, or postoperative bleeding that resulted in a return trip to the operating room. In contrast to the study by Shah and colleagues,

TABLE 2. Intraoperative and pathologic results from patients undergoing left upper lobectomy after previous coronary artery bypass grafting

\begin{tabular}{lc}
\hline \multicolumn{1}{c}{ Approach } & Value \\
\hline Planned thoracotomy & $25(89.3)$ \\
Robotics converted to thoracotomy & $2(7.1)$ \\
Robotics & $1(3.6)$ \\
Presence of adhesions from left upper lobe to graft & $26(92.9)$ \\
Left upper lobe completely mobilized off grafts & $25(89.3)$ \\
No LIMA graft & $1(3.6)$ \\
Wedge of lung left on graft & $2(7.1)$ \\
LIMA injury & $0(0)$ \\
Blood transfusion & $3(11.1)$ \\
Curative resection & $28(100)$ \\
Lymph nodes resected & $12.0 \pm 6.3$ \\
NSCLC & $26(92.9)$ \\
Stage 1 NSCLC & $16(57.1)$ \\
Stage 2 NSCLC & $3(10.7)$ \\
Stage 3 NSCLC & $5(17.9)$ \\
Squamous cell carcinoma & $15(53.6)$ \\
Adenocarcinoma & $6(21.4)$ \\
Other & $4(14.3)$ \\
Metastatic non-lung cancer & $2(7.1)$ \\
Renal cell carcinoma & $2(7.1)$ \\
\hline
\end{tabular}

Values are presented as raw number (\%), or mean \pm SD. For intraoperative blood transfusion, data were available on 27 patients. LIMA, Left internal mammary artery; $N S C L C$, non-small cell lung cancer.
TABLE 3. Postoperative outcomes of patients undergoing left upper lobectomy after previous coronary artery bypass grafting

\begin{tabular}{lc}
\hline \multicolumn{1}{c}{ Outcome } & Value \\
\hline Length of stay (d) & $4 \pm 2.1$ \\
Morbidity & $12(42.9)$ \\
Any & $5(17.9)$ \\
Prolonged air leak or subcutaneous emphysema & $5(17.9)$ \\
Atrial fibrillation & $1(3.6)$ \\
DVT & $1(3.6)$ \\
UTI & $1(3.6)$ \\
Loculated effusion requiring reoperation & $0(0)$ \\
Hemorrhage requiring reoperation & $0(0)$ \\
Myocardial infarction & $0(0)$ \\
Pneumonia & \\
ICU admission & $4(14.3)$ \\
Planned & $1(3.8)$ \\
Unplanned & $0(0)$ \\
30-d mortality & $0(0)$ \\
$90-d$ mortality &
\end{tabular}

Values are presented as raw number $(\%)$, or mean \pm SD. $D V T$, Deep vein thrombosis; $U T I$, urinary tract infection; $I C U$, intensive care unit.

the large majority of the patients in this study underwent lobectomy via a planned thoracotomy. Despite this fact, we demonstrated a length of stay (median of 4 days in both) and morbidity $(42.9 \%$ in this study vs $35.7 \%$ in their study) similar to those in their series of patients who underwent video-assisted thoracic surgery (VATS) lobectomy.

Shah and colleagues ${ }^{1}$ found a trend toward increased cardiac complications in their previous CABG group, driven mostly by the increased rate of atrial fibrillation (35.7\% in the LIMA group vs $16.3 \%$ in the control group). In our series, the risk of atrial fibrillation in patients undergoing left upper lobectomy after CABG was about $18 \%$, which is similar to rates of atrial fibrillation reported in series of open and minimally invasive lobectomies, which range from $12 \%$ to $16 \%{ }^{6-8}$ Evidence is conflicting regarding the significance of coronary artery disease as a risk factor for atrial fibrillation after pulmonary surgery. ${ }^{9}$

For preoperative studies and operative planning, we obtain a stress test as part of our standard protocol in patients undergoing lobectomy. If the stress test is abnormal, we perform a contrast arteriogram with interrogation of the grafts. However, we do not routinely perform a coronary arteriogram in these patients.

Up to $25 \%$ of vein grafts and $5 \%$ of arterial grafts are occluded at 5 years after an operation. ${ }^{10}$ Given the poor patency of vein grafts $(50 \%$ show evidence of disease by 5 years), we believe that performing a stress test before lobectomy is a reasonable approach if the patient has had a history of CABG, even if they are asymptomatic. More than $20 \%$ of the patients who received a stress test had findings suggestive of ischemia that warranted a left heart catheterization. 


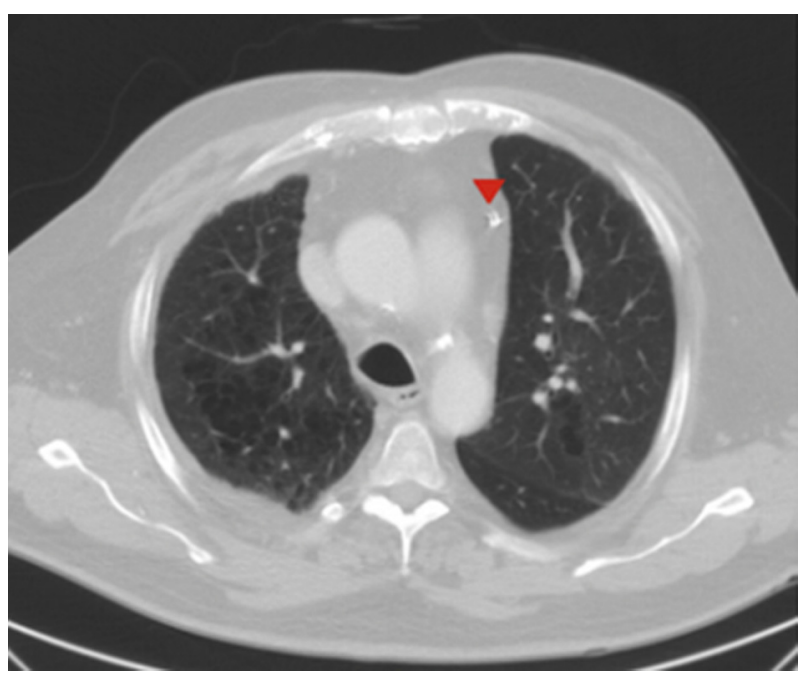

FIGURE 1. Computed tomography scan with intravenous contrast, demonstrating position of the left internal mammary artery graft (red arrow).

On the other hand, given that $85 \%$ to $95 \%$ of patients have a patent LIMA 10 years after transplant, we assume that the LIMA graft is contributing to myocardial blood flow (ie, that all of the patients are graft dependent) and give it corresponding respect when operating nearby. ${ }^{11}$ Reviewing the computed tomography scan may be helpful for tracking the course of the LIMA pedicle, either by identifying clips along the mammary, or if intravenous contrast is given, visualizing the LIMA itself (Figure 1). The prior operative report from CABG is desirable to have as well, to determine if the LIMA pedicle was used. Bypass grafts other than the LIMA may be vulnerable during dissection toward the medial part of the chest cavity.

With regard to intraoperative conduct, patients currently undergoing pulmonary lobectomy at our institution do not typically receive an arterial line, central line, or Foley catheter. This study demonstrates that we can perform the surgery safely in even those patients with previous $\mathrm{CABG}$, without the need of these invasive monitoring devices. None of the patients had a transesophageal echocardiogram probe placed. We acknowledge that intraoperative injury to the LIMA, although rare, is likely to have significant hemodynamic consequences for the patient. We therefore recommend that surgeons use the monitoring devices that they are comfortable with and typically use during pulmonary lobectomy.

Once surgery has commenced, we are prepared to nearly always encounter adhesions between the lung and the pedicle of the graft. We favor starting in the inferior anterior aspect of the lingula followed by hugging the lung until the clips on the mammary artery graft are identified. Only 1 of 27 patients who had LIMA grafting $(3.7 \%)$ did not have any adhesions of the lung to the graft in our study. These adhesions are generally encountered along the medial aspect of the left upper lobe to the mediastinum and can run toward the apex of the chest cavity, where the LIMA originates from the subclavian artery. In addition, we often encountered adhesions of the lower lobe to the surrounding chest wall, presumably owing to violation of the pleural space during LIMA harvesting. These adhesions were divided to completely free the lung, which helps us achieve a satisfactory N2 lymph node dissection and optimize pleural surface apposition.

Although we did not have any internal mammary artery injuries in our patients, we recommend immediate heparin administration and an attempt to reestablish flow through the graft if injury does occur. Temporary atraumatic clamps may need to be applied around the injury to allow either repair or more-extensive reconstruction of the conduit. If hemodynamic instability occurs that does not respond to vasoactive and/or inotropic medications, cardiopulmonary bypass can be initiated, either through the femoral vessels or via the aortic arch (arterial outflow) and pulmonary artery (venous inflow) in the left chest. Flow probes can be used to confirm patency of the graft after repair. Postoperative coronary catheterization may be necessary on a selected basis.

We should emphasize that $>90 \%$ of our patients underwent thoracotomy, and that these results may not be generalizable to surgeons seeking to pursue a minimally invasive approach for these patients. For patients undergoing video-assisted thoracic surgery lobectomy, the strategy of leaving a small rim of parenchyma near the bypass graft, as recommended by Shah and colleagues, may be wiser from a safety perspective. However, this method has hypothetical oncologic disadvantages. Shah and colleagues ${ }^{1}$ showed increased cancer recurrence rates of patients in their CABG group, compared with their control group ( $27 \%$ vs $15 \%$ in patients of all stages, $30 \%$ vs $10 \%$ in patients with stage 1 lung cancer), although these findings did not reach statistical significance $(P=.27$ and $P=.051$, respectively). In patients with tumor abutting or near the LIMA graft, converting to a thoracotomy may be preferred to ensure complete resection. Nonetheless, we acknowledge that because of the size of our study and the fact that we had no local recurrences among these patients, we could not prove that complete removal of the left upper lobe from the graft had an oncologic benefit compared with leaving a sliver of lung.

In this series, we attempted robotics-assisted lobectomy for 3 patients, but we were only able to complete the operation using robotics for 1 of them. Of the 2 patients that were converted to a thoracotomy, 1 had a tumor involving the base of a lingular artery, necessitating pulmonary artery resection and arterioplasty, and the other had an injury to an anteroapical trunk off of the left main artery requiring repair. In neither case were adhesions 
from the bypass graft to the left upper lobe the primary reason for conversion; all 3 had the left upper lobe dissected off the bypass grafts before opening.

Although our study did not attempt to address this issue, we think that the optics and dexterity of robotics techniques may actually be beneficial for surgeons who are trying to dissect the left upper lobe off the bypass grafts with a minimally invasive approach. At this point, the senior investigator of this study generally prefers to do these cases using robotics. We recommend that surgeons use the technique they are most comfortable with; this study specifically demonstrates the safety of thoracotomy when operating on this particular patient population.

Limitations of this study include the fact that it was a single-surgeon series performed at an academic institution, which may limit the generalizability of our conclusions about safety. As Shah and colleagues ${ }^{1}$ allude to in their discussion, having an immediate cardiac surgery backup in case of a graft injury may increase the comfort level and advisability of performing left upper lobectomy after CABG in centers where this scenario is less common. We did not obtain postoperative cardiac enzymes and electrocardiograms unless indicated by the patient's clinical status, which may have limited the sensitivity of detecting myocardial ischemia and/or infarction.

In conclusion, we have shown that previous CABG is not a contraindication to left upper lobectomy. If careful dissection is used, morbidity or 30- or 90-day mortality does not seem to increase. Although previous CABG results in significant adhesions between the lung and the LIMA graft, meticulous dissection of the left upper lobe off the graft allows for a left upper lobectomy to be performed, with complete removal of the lobe, without injury to the grafts, and with minimal perioperative cardiac complications.

\section{Conflict of Interest Statement}

Robert J. Cerfolio reports other from Intuitive surgical, other from Ethicon, and other from Community Health Systems, outside the submitted work. The other authors have nothing to disclose with regard to commercial support.

\section{References}

1. Shah AA, Worni M, Onaitis MW, Balderson SS, Harpole DH, D'Amico TA, et al. Thoracoscopic left upper lobectomy in patients with internal mammary artery coronary bypass grafts. Ann Thorac Surg. 2014;98:1207-12.

2. Funaki S, Inoue M, Shigemura N, Okumura N. Thoracoscopic lobectomy for lung cancer after coronary artery bypass grafting using internal thoracic artery Interact Cardiovasc Thorac Surg. 2012;15:928-9.

3. Halkos ME, Sherman AJ, Miller JI Jr. Preservation of the LIMA pedicle after cardiac surgery in left upper lobectomy. Ann Thorac Surg. 2003;76:280-1.

4. Cerfolio RJ, Bryant AS, Skylizard L, Minnich DJ. Initial consecutive experience of completely portal robotic pulmonary resection with 4 arms. J Thorac Cardiovasc Surg. 2011;142:740-6.

5. Santini M, Fiorello A, Vicidomini G, Laperuta P. The use of LigaSure for preservation of a previous coronary artery bypass graft by using the left internal thoracic artery in a left upper lobectomy. J Thorac Cardiovasc Surg. 2008;136: 222-3.

6. Vaporciyan AA, Correa AM, Rice DC, Roth JA, Smythe WR, Swisher SG, et al Risk factors associated with atrial fibrillation after noncardiac thoracic surgery: analysis of 2588 patients. J Thorac Cardiovasc Surg. 2004;127:779-86.

7. Berry MF, D'Amico TA, Onaitis MW. Use of amiodarone after major lung resection. Ann Thorac Surg. 2014;98:1199-206.

8. Onaitis M, D'Amico T, Zhao Y, O'Brien S, Harpole D. Risk factors for atrial fibrillation after lung cancer surgery: analysis of the Society of Thoracic Surgeons general thoracic surgery database. Ann Thorac Surg. 2010;90:368-74.

9. Ivanovic J, Maziak DE, Ramzan S, McGuire AL, Villeneuve PJ, Gilbert S, et al Incidence, severity, and perioperative risk factors for atrial fibrillation following pulmonary resection. Interact Cardiovasc Thorac Surg. 2014;18:340-6.

10. Fitzgibbon GM, Kafka HP, Leach AJ, Keon WJ, Hooper GD, Burton JR. Coronary bypass graft fate and patient outcome: angiographic follow-up of 5,065 grafts related to survival and reoperation in 1,388 patients during 25 years. $J$ Am Coll Cardiol. 1996;28:616-26.

11. Goldman S, Zadina K, Moritz T, Ovitt T, Sethi G, Copeland JG, et al. Long-term patency of saphenous vein and left internal mammary grafts after coronary artery bypass surgery: results from a Department of Veterans Affairs Cooperative Study. J Am Coll Cardiol. 2004;44:2149-56.

Key Words: Coronary artery bypass grafting, left upper lobectomy, internal mammary artery 